\title{
Bacteroides faecis
}

National Cancer Institute

\section{Source}

National Cancer Institute. Bacteroides faecis. NCI Thesaurus. Code C111134.

A species of anaerobic, gram-negative, rod shaped bacteria assigned to the phylum Bacteroidetes. This species is non-motile, spore forming, oxidase, urease and catalase negative, indole positive and hydrolyzes esculin. B. faecis was originally isolated from human feces. 